\title{
„ICH HABE [...] KEINE SONATE SCHREIBEN WOLLEN, SONDERN EINEN ROMAN“ - ZU AUTOREFERENZIELLEN ASPEKTEN VON THOMAS MANNS DOKTOR FAUSTUS
}

\begin{abstract}
I wanted [...] to write a novel, not a sonata“ - on autoreferential aspects of Thomas Mann's Doktor Faustus

In the centre of the analysis stands the autoreferential dimension of Thomas Mann's Doktor Faustus and its relation to the novel's socio-philosophical message, which, although not free from ironical overtones, tends to be a didactic plea for humanism. However, the main emphasis of the investigation is not the humanist idea itself but, above all, the narrative methods used to infuse this idea with new means of expression. Therefore, the music-motif in the novel is interpreted as a metaphor of an intertextual literary motif. From a metaliterary perspective, the main question in Mann's partly consistent, yet partly paradoxical art- and soul-study is the following: how can (and should) the Faust-legend be reshaped and interpreted to match the demands of the modern age? The attempt to discuss the ,national myth ' in such a new way is made by three authorities: two fictional and one real. The protagonist, Adrian Leverkühn, the first-person narrator, Serenus Zeitblom, and, finally, the author himself, Thomas Mann, each in turn, whether wilfully or unconsciously, offers their own art-concept. To a great extent, the general semantic picture of the story told in Faustus is determined by various distinctions between the intratextual ideological confrontation ,Leverkühn vs. Zeitblom ' and the extratextual outside-perspective of the author, who stays paramount to the novel-figures. My article attends to the narratological as well as the philosophical decoding of this overall-picture.
\end{abstract}

\section{Einleitung}

Der vorliegende Beitrag bezieht sich auf die autoreferenzielle Dimension von Thomas Manns Roman Doktor Faustus und deren Verhältnis zur semantischen Ebene des Textes.

Im ersten Kapitel wird daher der Begriff der Autoreferenzialität und ihre Rolle in der Literatur zunächst kurz erläutert. Dabei wird die Selbstreferenz eines literarischen Textes im Sinne von Jean-Philippe Jacquard verstanden, dessen Ansichten zu diesem Thema zusammenfassend dargestellt werden. Dies ist insofern wichtig, als eine Analyse des Musik- und Literaturmotivs in Doktor Faustus, die die autorefe- 
renzielle Perspektive nicht beachtet, einer interpretatorischen Vereinfachung leicht verfallen kann.

Das zweite Kapitel befasst sich mit der narrativen Struktur des Romans. Im Mittelpunkt der Untersuchungen steht dabei „die eigenartige Erzählsituation des Faustusromans " ${ }^{\text {1 }}$, deren komplizierter Aufbau die Figur des Protagonisten Adrian Leverkühn im Vexierspiegel Serenus Zeitbloms Erinnerungen wiedergibt ${ }^{2}$.

Schließlich wird im dritten, resümierenden Kapitel der Frage nachgegangen, inwiefern man Doktor Faustus als eine modernisierte und humanisierte Version der Faustlegende deuten kann, d.h., was, wie und zu welchem Zweck modernisiert wird.

Bei einem zehnseitigen Artikel über einen thematisch derartig umfangreichen und polyphonen Roman wie Thomas Manns Doktor Faustus - einen Roman mit einer beinahe hundertjahrelangen Rezeptionsgeschichte - steht man immer wieder vor dem Problem der Selektion von Sekundärliteratur. Den Ausgangspunkt meiner Betrachtungen bildet vor allem der Quellentext. Der Grund dafür ist, dass das Autoreferenzielle des analysierten Romans eine sehr bescheidene, fast unbeachtete Stelle im literaturwissenschaftlichen Faustus-Diskurs einnimmt. Auf die, wenn nicht immer einseitige, so doch ziemlich homogene Forschungstradition, die den Roman als eine Art Abrechnung Manns mit dem nationalsozialistischen Deutschland interpretiert, wurde bereits 1969 in Hubert Orłowskis Prädestination des Dämonischen, einer fundamentalen Studie zu den strukturellen Besonderheiten von Doktor Faustus, hingewiesen:

\begin{abstract}
Was sich fast bei allen Kritikern wiederholt, ist ein In-Beziehung-Bringen des Schicksalsweges Adrian Leverkühns und des der deutschen Nation sowie der Betonung des antihumanistischen Charakters des musikalischen Schaffens des Tonsetzers. [...] Der besonderen Erzählsituation des Romans resp. der Person Serenus Zeitbloms in der Rolle des Erzählers wird keine Beachtung geschenkt. ${ }^{3}$
\end{abstract}

Zwar wurde die Faustus-Rezeption in literaturwissenschaftlichen Kreisen seither durch mehrere neue Beiträge bereichert, aber die allgemeine Denk- und Schlussfolgerungsrichtung bleibt dem von Orłowski exakt formulierten (und bemängelten) Deutungsschema erstaunlich treu. Orłowskis präziser, mehrere narrative wie philosophische Aspekte beleuchtender Forschungsbeitrag zum strukturellen Gerüst von Thomas Manns Altersroman hat, die erzähltechnische Mehrdeutigkeit des Textes

\footnotetext{
${ }^{1}$ Hubert Orłowski: Prädestination des Dämonischen. Zur Frage des bürgerlichen Humanismus in Thomas Manns ,Doktor Faustus “, Poznan 1969.

${ }^{2}$ Siehe dazu Hermsdorfs interessante, wenn auch (was die angesprochene Distanzlosigkeit betrifft) etwas zu weit gehende Bemerkung: „Mit der Rahmenhandlung des „Narrators“ erreicht der Roman eine reportagenhaft direkte Zeitnähe, die sich in Thomas Manns Schaffen niemals wiederfindet und selbst in der Geschichte der modernen Erzählprosa ungewöhnlich ist. Nichts von epischer Distanz", in: Peter Fix, Ulla Fix, Klaus Hermsdorf, Fritz Hofmann, Fritz Henry Matter, Konrad Paul: Das erzählerische Werk Thomas Manns, Berlin/Weimar 1976, S. 295.

${ }^{3}$ Ebd., S. 15.
} 
betonend, überzeugend bewiesen, wie eng die Form von Doktor Faustus mit seinem Inhalt (nicht im Sinne der Handlung, sondern der noetischen Aussage) verknüpft ist. Ohne eine aus rein praktischen Gründen unmögliche allumfassende und allberücksichtigende Analyse anzustreben, habe ich in meinem Artikel gewagt, einige Thesen zum Thema der autoreferenziellen Dimension des Romans und zu deren Einfluss auf die Modernisierung der Faustgeschichte aufzustellen.

\section{Musik und Literatur: die funktionale Autoreferenzialität von Doktor Faustus}

Unter dem Begriff der Autoreferenzialität versteht man in der Literaturwissenschaft die Selbstbezüglichkeit eines literarischen Werkes. Diese Bezeichnung wird in der Regel bei der Analyse von postmodernistischen Texten (oder deren Vorläufern wie z.B. Lawrence Sternes Tristram Shandy) verwendet. Wie der französische Slawist Jean-Philippe Jacquard zu Recht feststellt, bezieht sich die autoreferenzielle Dimension jedoch nicht nur auf die verwickelten Literaturrätsel der Neuzeit, sondern vielmehr auf die literarische Kunst als solche:

Jedes Werk, wie spannend und hochwertig sein erzählerischer Inhalt auch sein mag, spricht von sich selbst [...], mehr oder weniger keusch oder, im Gegenteil, kokettierend, mit seinen zahlreichen Spiegeln spielend, einen Text in den anderen endlos implizierend.

Dieses Widerspiegelungsspiel ist im Zeitalter des „Realismus“ schwerer und in anderen Epochen leichter zu erkennen [...], aber in der Tat wird es stets getrieben, denn ohne dieses Spiel gäbe es einfach keine Literatur. ${ }^{4}$

In seinen Untersuchungen belegt Jacquard diese These an mehreren Beispielen aus der russischen Klassik (u.a. Puschkin und Gogol), sodass die angeborene autoreferenzielle Funktion der Literatur schließlich als eine unumstrittene Tatsache erscheint. Unabhängig davon, ob man Jacquards Totalität in Bezug auf die literarische Autoreferenzialität teilt, gehört Thomas Mann zweifellos zu denjenigen Autoren, deren Romane durch einen hohen Grad an Selbstreferenz gekennzeichnet sind. Umso erstaunlicher ist es, dass gerade in Doktor Faustus ein höchstbedeutender Aspekt dieses bei Mann so oft auftretenden Kunstgriffs von der Forschung meist übersehen wird. Es geht nämlich um das Verhältnis zwischen dem musikalischen und dem literarischen Motiv des Romans. Darunter sollte man aber keineswegs die mehrmals betonte

\footnotetext{
${ }^{4}$ Jean-Philippe Jacquard: Literatura kak takovaja [Literatur als solche]. Novoe Literaturnoe Obozrenie: Moskva 2011, S. 11: „Всякое произведение, как бы увлекательно и добротно ни было его повествовательное содержание, говорит о себе самом [...], проявляя при этом большее или меньшее целомудрие или, наоборот, кокетство, играя со своими многочисленными зеркалами, без конца вкладывая текст в текст. Эта игра отражений менее различима в периоды «реализма» и более заметна в другие эпохи [...], а на самом деле она ведется всегда, потому что без нее литературы бы просто не было" [übersetzt von А.К.].
} 
und ohnehin ziemlich offensichtliche literatur-musikalische Symmetrie der Romanstruktur verstehen. Dass der Text selbst als eine Art Sinfonie mit polyphonischen Themen, montageartigen Kontrapunkten und einem grandiosen Finale aufgebaut ist, wurde mittlerweile zu einem beinahe klischeehaften Faktum. Allgemein greifen die Autoren, die sich mit Doktor Faustus auseinandersetzen, viel zu oft zu Musiktheorie bzw. Musikphilosophie, die dann in der Regel auf die globale Kultur- und Gesellschaftsproblematik übertragen wird (,das Versagen des modernen Humanismus“" ${ }^{\text {(5) }}$. Die Versuchung, Thomas Manns Roman aus dieser Perspektive zu analysieren, ist durchaus verständlich, steht ja die Musik im Zentrum des Textes. Paradoxerweise erscheint jedoch dieser Interpretationsweg nicht besonders fruchtbar, und zwar aus folgendem Grund. Der Text sagt zum Thema ,Musik` alles, was dazu gesagt werden soll. Daher wäre es aufschlussreicher, die Frage anders zu formulieren. Welche Bedeutung wird dem Musikthema im Roman beigemessen? Ist Doktor Faustus wirklich ein ,Musikroman' in dem Sinne, dass darin versucht wird, mit literarischen Mitteln von einer überwiegend nonverbalen Kunstgattung zu berichten? Verfolgt man den $\mathrm{Zu}-$ sammenhang von Musik und Literatur im Text etwas aufmerksamer, so lässt sich ein geradezu umgekehrtes Verhältnis beobachten. Michael Neumann macht hierzu eine interessante Bemerkung:

Beziehung ist alles. Und willst du sie näher bei Namen nennen, so ist ihr Name „Zweideutigkeit“, kurz: Musik sei „Zweideutigkeit als System“. ,Beziehung“ war auch Thomas Manns Schlüsselbegriff für die eigene, an Wagner orientierte leitmotivische Arbeit. ${ }^{6}$

Neumann entwickelt diesen zutreffenden Gedanken in der erprobten Tradition der oben erwähnten musikphilosophischen Interpretation. Es wird also über Ordnung und Freiheit, die Zweideutigkeit der Töne und Ähnliches reflektiert. Wie steht es aber um die Beziehung zwischen der beschriebenen und der betriebenen Kunst, d.h. zwischen Musik und Literatur? Beim näheren Hinblick wird sichtbar, wie eng diese Beziehung ist. Fangen wir mit einem eindeutigen Beispiel an. Als der Protagonist Adrian Leverkühn über eines seiner 1927 entstandenen Werke spricht, macht er eine erhellende Bekenntnis: „Ich habe“, sagte Adrian zu mir, „keine Sonate schreiben wollen, sondern einen Roman" ${ }^{67}$. Dabei geht es dem Komponisten sowohl um die strukturelle als auch um die inhaltliche Ebene seines Schaffens und deren Wirkung auf den Rezipienten (die für Adrian trotz seines aristokratischen Außenseitertums wichtig ist). Die Frage lautet daher: Wie würde derjenige „Roman“, den Adrian mit musikalischen Mitteln zu ,schreiben' versucht, in einer literarischen Form aussehen? Was wären seine Form und seine Aussage? Die Antwort liegt nahe, denn dieser Roman ist größtenteils Doktor Faustus. Man vergleiche allein schon den Stil

\footnotetext{
${ }^{5}$ Carl R. Stange: Thomas Manns „Doktor Faustus “, in: Literarische Revue, 1948, S. 378.

${ }^{6}$ Michael Neumann: Thomas Mann. Romane, Berlin 2001, S. 169.

${ }^{7}$ Thomas Mann: Doktor Faustus, 2. Aufl., Berlin/Weimar 1975, S. 620.
} 
sowie den Inhalt des Textes mit Adrians Musikwahrnehmung, die er im Brief an seinen Lehrer Wendell Kretzschmar zum Ausdruck bringt:

Mein Freund, warum muß ich lachen? Kann man mit mehr Genie das Hergebrachte benutzen, die Kniffe weihen? Kann man mit gewiegterem Gefühl das Schöne erzielen? Und ich Verworfener muß lachen, namentlich bei den grunzenden Stütztönen des Bombardons - Wum, wum, wum Pang! -, ich habe vielleicht zugleich Tränen in den Augen, aber der Lachreiz ist übermächtig, - ich habe verdammterweise von jeher bei den geheimnisvoll-eindrucksvollsten Erscheinungen lachen müssen und bin von diesem übertriebenen Sinn für das Komische in die Theologie geflohen, in der Hoffnung, daß sie dem Kitzel Ruhe gebieten werde, - um dann eine Menge entsetzlicher Komik in ihr zu finden. Warum müssen fast alle Dinge mir als ihre eigene Parodie erscheinen? Warum muß es mir vorkommen, als ob fast alle, nein, alle Mittel und Konvenienzen der Kunst heute nur noch zur Parodie taugten? ${ }^{8}$

Das Schlüsselwort „Parodie“, das Thomas Mann (durch die transparente Vermittlung von Serenus Zeitblom) für seine Leser mit sorgsamer Kursivschrift hervorhebt, betrifft selbstverständlich im gleichen Maße Leverkühns Musikstücke und Zeitbloms mal übertrieben pathetische, mal vernichtend ironische Anmerkungen über Kunst, Gott, Liebe, Vaterland und andere ,große Themen'. Um es mit Erich Hellers ausführlicher Formulierung auf den Punkt zu bringen:

Ja, die Inkommensurabilität des Gegenstands mit der Literatur in jedweder Gestalt, und also die Unmöglichkeit, ein hocherhobenes, aber dennoch typisches Leben der Epoche mit den Mitteln der Kunst zu bewältigen, ist das formbestimmende Thema dieses ,unmöglichen“ Werks. ${ }^{9}$

Mit anderen Worten ist das „formbestimmende Thema“ von Doktor Faustus die unüberwindbare Diskrepanz zwischen dem gehobenen literarischen Stil und der wahrheitsgetreuen Epochenschilderung. Sucht man aber nach dem Sinn bestimmenden Thema, so trägt es stark ausgedrückte autoreferenzielle Züge, worauf allein schon der Titel des Romans verweist. So betrachtet ist Doktor Faustus ein sich als Roman verstellender literaturtheoretischer Versuch, der alten Faust-Geschichte (als deutschem Nationalmythos) eine zeitgenössische Rechnung zu tragen. Möchte man die semantische Dimension dieser modernisierten Legende tiefer erforschen, so ist der weitere relevante Bezugspunkt die narrative Trinität des Textes.

\section{Der dreifache Spiegel: Protagonist - Erzähler - Autor}

Hans Mayer behauptet, die Figur des Erzählers Serenus Zeitblom gebe dem Autor von Doktor Faustus ,die Chance, den Roman auf drei Ebenen zu führen: als

\footnotetext{
${ }^{8}$ Ebd., S. 182-183.

${ }^{9}$ Erich Heller: Thomas Mann. Der ironische Deutsche, Frankfurt a.M. 1959, S. 307.
} 
Lebensgeschichte Leverkühns, als Entstehungszeit der von Zeitblom verfaßten Lebensgeschichte Leverkühns - und als Entstehungsgeschichte des von Thomas Mann verfaßten Faustusromans $[\ldots]^{\star 10}$. Daraus folgt, dass sich auch die narrative Struktur des Romans aus drei Perspektiven betrachten lässt. „Die Frage, wie sich die Beziehung zwischen Thomas Mann und seiner Erzählerfigur Zeitblom darstellt, spielt in der umfangreichen Forschungsliteratur zum ,Doktor Faustus' eine erstaunlich geringe Rolle“11, postulierte Hans Hilgers 1995 und berührte damit einen wesentlichen Textfaktor. Hilgers erforscht diese Beziehung hauptsächlich aus dem sozialpolitischen Blickwinkel, sich auf das kritisch-patriotische Deutschland-Verhältnis von Zeitblom und Mann konzentrierend. In meinem Artikel werde ich diesen Aspekt nicht beleuchten und den Fokus auf die narrative Konkurrenz ,Erzähler vs. Autor übertragen. Bereits im ersten Kapitel räumt der Erzähler Serenus Zeitblom nach einigen einführenden Überlegungen über Kunst und Genie eine vorzeitige Enthüllung des zentralen Romanthemas ein:

Ich hatte soeben kaum die Feder angesetzt, als ihr ein Wort entfloß, das mich heimlich bereits in eine gewisse Verlegenheit versetzte: das Wort „genial“ [...]. Nun ist dieses Wort „Genie“, wenn auch über-mäßigen, so doch gewiß edlen, harmonischen und human-gesunden Klanges und Charakters [...]. Und doch ist nicht zu leugnen und ist nie geleugnet worden, daß immer eine leises Grauen erweckende Verbindung besteht zwischen ihr und dem unteren Reich, und daß eben darum die versicherten Epitheta, die ich ihr beizulegen versuchte, „edel“, „human-gesund“ und „harmonisch“, nicht recht darauf passen wollen [...].

Hier breche ich ab, mit dem beschämenden Gefühl artistischer Verfehlung und Unbeherrschtheit. Adrian selbst hätte wohl kaum, nehmen wir an: in einer Symphonie, ein solches Thema so vorzeitig auftreten - hätte es höchstens auf eine fein versteckte und kaum schon greifbare Art von ferne sich anmelden lassen. ${ }^{12}$

Dass Zeitblom seine Notizen mit einer Sinfonie vergleicht, ist natürlich nicht zufällig und bringt den Leser gleich auf den Gedanken, der ganze Roman sei als eine ,literarische Sinfonie' strukturiert. Wie gesagt ist diese Schlussfolgerung zu offensichtlich, um hier weiterentwickelt zu werden. Viel spannender ist das latente Spiel, das von der dem Erzähler übergeordneten Autoreninstanz getrieben wird. (Die Foucault-verpflichtete Skepsis vorwegnehmend sei hier betont, dass unter dem Autor Thomas Mann der Verfasser des Romans und nicht etwa der Mensch bzw. eine historische Figur verstanden wird). Zeitblom behauptet ja, Adrian würde sein Werk kunstfertiger gestalten und den Grundgedanken erst später oder, wenn schon am Anfang, dann wenigstens in einer verhüllten Form äußern. Alles hängt allerdings davon ab, was genau man als den Grundgedanken von Doktor Faustus bezeichnet. Zeitblom setzt dafür die paradoxal-dualistische Natur des künstlerischen Genies an.

\footnotetext{
${ }^{10}$ Hans Mayer: Thomas Mann, Berlin 1949, S. 378-379.

${ }^{11}$ Hans Hilgers: Serenus Zeitblom: der Erzähler als Romanfigur in Thomas Manns „Doktor Faustus", Frankfurt a.M. 1995, S. 103.

${ }^{12}$ Mann: Doktor Faustus, S. 8-9.
} 
Der nicht verlautbarten Übereinkunft zwischen dem Leser und dem Erzähler demütig gehorchend stimmen sämtliche Mann-Forscher mit Zeitbloms Behauptung überein. So wurde das scheinbar unbestrittene Fazit zusammengefasst, das Hauptthema des Romans sei diese tragische Dichotomie der großen Kunst, die Adrians Biograph in seinen weitschweifigen sozialphilosophischen Monologen so häufig anspricht. Kurz: bleibt man auf der Ebene der Erzählerwelt, so ist das vom Narrator formulierte Thema des Romans zweifellos zentral; verlässt man aber diese Ebene, um sich über den Zeitblom-Raum zu erheben und den ganzen Text aus einer distanzierten Außenperspektive zu betrachten, so eröffnen sich gleich mehrere narrative Einzelheiten, die auf eine alternative Lösung hinweisen. Zeitblom meint, Leverkühn würde das Hauptthema ,auf eine fein versteckte und kaum schon greifbare Art“ erscheinen lassen. Da Zeitblom jedoch nur der fiktive Erzähler und nicht der Autor von Doktor Faustus ist, wäre es logisch, seine Aussage zu hinterfragen und festzustellen, ob vielleicht eben die angeführte Textpassage eine „fein versteckte und kaum schon greifbare" Andeutung enthält, nämlich die Andeutung auf die autoreferenzielle Semantik des Romans. Kehren wir zurück zur oben aufgestellten These, Doktor Faustus sei ein Gedankenexperiment darüber, wie die moderne Auffassung der Faust-Legende aussehen könnte. Nehmen wir demnächst an, eine primäre Voraussetzung für die Modernisierung der klassischen Faust-Geschichte wäre die ironische Doppelbödigkeit, die spielerische Mehrdeutigkeit der Neuzeit. Man könnte diese Botschaft dann freilich kaum feiner und unangreifbarer verstecken, als sie unter Zeitbloms trügerischer Selbstskepsis zu begraben. Das parallele Hauptthema des Romans - seine multidimensionale Autoreferenzialität - deutet sich damit schon am Anfang des Textes an, und zwar in der besten Tradition von Adrian Leverkühns Sinfonien: leise, anonym und nahezu unbemerkbar. (In diesem Sinn erscheint Thomas Mann in seinem Roman nicht etwa, wie Georg Lukács geglaubt hat, als „Spiegel der Welt‘،13, sondern als ,Spiegel des Spiegels“.)

Dass Serenus Zeitblom allgemein kein zuverlässiger Erzähler ist, wurde von Orłowski mithilfe mehrerer Textauszüge veranschaulicht und belegt. „Das Bild, welches der Leser von Adrian erhält", stellt Orłowski nach einer Reihe ausführlicher Beweise fest, ,ist durch Zeitbloms hineinprojizierte Wunschdeutungen und -vorstellungen natürlich deformiert “14 . Dies betrifft allerdings nicht nur die Gestalt Leverkühns, sondern durchaus auch Zeitbloms Selbstschilderung als Erzählinstanz. So versichert er dem Leser ab und zu, seine Notizen haben mit der literarischen Kunst nichts zu tun, indem er zum Beispiel sagt: „Nochmals, ich schreibe keinen Roman und spiegle nicht allwissende Autoreneinsicht in die dramatischen Phasen einer intimen, den Augen der Welt entzogenen Entwicklung vor.“15 Das stimmt

\footnotetext{
${ }^{13}$ Zitiert nach: Eva Schmidt-Schütz: ,Doktor Faustus “ zwischen Tradition und Moderne, Frankfurt a.M. 2003, S. 298.

${ }^{14}$ Orłowski: Prädestination des Dämonischen, S. 116.

${ }^{15}$ Mann: Doktor Faustus, S. 450.
} 
allerdings, denn den Roman schreibt in der Tat nicht Serenus Zeitblom, sondern Thomas Mann. Deshalb widerspricht der Erzähler einige Kapitel später seinen eigenen Worten mit einer hinterhältig-verzweifelten Exklamation:

Wie viele Schriftsteller vor mir schon mögen die Untauglichkeit der Sprache beseufzt haben, Sichtbarkeit zu erreichen, ein wirklich genaues Bild des Individuellen hervorzubringen! Das Wort ist geschaffen für Lob und Preis, es ist ihm verliehen zu erstaunen, zu bewundern, zu segnen und die Erscheinung durch das Gefühl zu kennzeichnen, das sie erregt, aber nicht, sie zu beschwören und wiederzugeben. ${ }^{16}$

Hier spricht schon evident die Stimme des Autors durch die des Erzählers, weil Zeitblom ja kein Schriftsteller ist. Mehr noch: Die dem Erzähler zugeschriebene Klage über die begrenzten Möglichkeiten der literarischen Sprache ist fast ein Zitat einer der (aus erzähltechnischer Sicht) interessantesten Stellen aus Gustav Flauberts Madame Bovary, wo der Narrator die Gedanken von Rodolf, Emmas zynischem Liebhaber, folgendermaßen kommentiert:

Er hatte sie all das so oft sagen hören, daß es für ihn nichts Originelles mehr hatte. [...] Weil ihm leichtfertige oder käufliche Lippen solche Sätze zugeflüstert hatten, glaubte er nur wenig an die Aufrichtigkeit der jetzigen; man sollte, dachte er, das überspannte Gerede, das mittelmäßige Leidenschaften verbirgt, dabei unterlassen: als quölle die übervolle Seele nicht manchmal in hohlsten Metaphern über, denn niemand kann je seine Bedürfnisse, seine Vorstellungen, seine Schmerzen so äußern, wie sie wirklich sind, und die menschliche Sprache ist ein verstimmter Klimperkasten, auf dem wir Melodien spielen, zu denen man die Bären tanzen lassen könnte, obgleich man die Sterne rühren wollte. ${ }^{17}$

Der Erzähler macht hier einen plötzlichen Übergang von der auktorialen Gedankendarstellung der Figur zur personalen Auseinandersetzung mit dem Problem der professionellen Schwierigkeiten des künstlerischen Schrifttums bei Gefühlsdarstellung ${ }^{18}$. Immerhin scheinen die Parallelen unübersehbar zu sein. Thomas Manns latentes Spiel mit Zitaten und narrativen Instanzen sollte jedoch von den intertextuellen Experimenten des Postmodernismus klar abgegrenzt werden, was in der neuesten Rezeption nicht immer der Fall ist, worauf Eva Schmidt-Schütz in ihrer eingehenden Forschung zu diesem Thema hinweist: „In jüngster Zeit schienen sich die Koordinaten sogar gänzlich zu verschieben, Doktor Faustus, Thomas Manns großer Altersroman, wurde neu entdeckt und plötzlich als Werk der Postmoderne gelesen. ${ }^{“ 19} \mathrm{Ob}$ man dabei von einer Neuentdeckung oder einem Missverständnis sprechen sollte, bleibt jedoch zumindest strittig. Die Autoreferenzialität an sich ist in Doktor Faustus kein Selbstziel, denn Thomas Mann gehört zu den Autoren, in deren Werken jedes

\footnotetext{
${ }^{16}$ Ebd., S. 626.

${ }^{17}$ Gustav Flaubert: Madam Bovary, Berlin 1980, S. 212.

${ }^{18}$ Vgl. Vladimir Nabokov: Lekcii po zarubezhnoj literature [Vorlesungen über die fremdsprachige Literatur], Sankt-Petersburg 2011, S. 253.

${ }^{19}$ Schmidt-Schütz: ,Doktor Faustus “ zwischen Tradition und Moderne, S. 15.
} 
Mittel einem bestimmten Zweck dient. Außerdem bekommt dieser Zweck in seinem Fall stets eine soziokulturelle Färbung. Letztere wird im weiteren Teil der vorliegenden Analyse etwas näher betrachtet.

\section{Zwischen Klassik und Moderne: die Wege der Kunst}

Wir haben bisher konstatiert, Thomas Manns Doktor Faustus sei kein traditioneller Roman im Sinne einer spannend erzählten, aktionsreichen Geschichte, sondern eher eine künstlerisch gestaltete Antwort auf die Frage nach einer zeitgenössischen Version der Faust-Legende. (Es handelt sich hier sowie um den für den Autor zeitgenössischen Gesellschafts- und Kulturraum, also die Kriegs- und Nachkriegsepoche, in welcher der Text entstanden ist, als auch um die fiktiv-literarische Umgestaltung dieses Raumes im Roman). Heute würde man sagen, Mann habe den FaustDiskurs um eine neue Darstellungsfacette erweitert, bei der der autoreferenzielle Aspekt eine höchstrelevante Rolle spielt. Nun wäre es sinnvoll, dem Ideengehalt des ,Faust-Remakes' Aufmerksamkeit zu schenken.

Nicht zu vergessen ist dabei Orłowskis überaus bedeutende Bemerkung, dass „,nicht die erzählte Geschichte an sich, sondern die Bezugnahme des Erzählers zu dem erzählten Stoffe, seine Vorliebe für bestimmte Details, das Auslassen anderer [...] die ,eigentliche“ Geschichte bilden“'20 würden. Der Pfad, der von der formalen zur inhaltlichen Ebene führt, ist stets der Auslegungsweg, auf den man sich am meisten verlassen kann.

Heller nennt Doktor Faustus eine, ,tragische Parodie des Frankfurter Volksbuchs aus dem Jahre $1587^{\text {، } 21}$. Diese Oxymoron-Definition ist äußerst treffend, und zwar aus doppelter Sicht. Einerseits wird das Tragische im Roman permanent in parodistischem Lichte betrachtet. Andererseits wird aber auch der Parodie ein obligatorisch tragischer Beigeschmack verliehen. Die ungeschriebenen „die Verbindung von Faust-Mythos, Nietzsche-Schicksal, fiktiver Biographie und Nationalgeschichte ${ }^{622}$ erlauben seine Texte mit einer reichlichen Portion parodistischer Elemente auszustatten. Pures Pathos funktioniert nicht mehr, klingt unglaubwürdig und wird vom Rezipienten entweder missverstanden oder verspottet. Je schöner und erhabener ein Musikwerk ist, desto lauter muss Adrian Leverkühn lachen, denn die Naivität der alten, klassischen Kunst entspricht nicht den grausamen Realien der nihilistischen Moderne. Aber Leverkühns und Manns Kunstauffassungen sind nicht identisch, deshalb kann man folgender These von Heller nur teilweise zustimmen:

\footnotetext{
${ }^{20}$ Orłowski: Prädestination des Dämonischen, S. 57.

${ }^{21}$ Heller: Thomas Mann. Der ironische Deutsche, S. 327.

${ }^{22}$ Eberhard Hilscher: Thomas Mann. Leben und Werk, Berlin 1983, S. 189.
} 
Wie Leverkühn die Neunte Symphonie ,zurücknimmt“, so gründlich nimmt Thomas Mann Goethes Faust zurück. [...] Goethes Faust [...] ist der poetische Höhepunkt jener ,harmonischen Subjektivität", in welcher die Seele geborgen ist, auch und gerade wenn sie sich müht und plagt [...] Leverkühns Seele entbehrt solcher Gewißheit. [...] So also vollzieht sich der Widerruf von Goethes Faust durch Thomas Manns Doktor Faustus. Denn hier spielt sich der Umgang zwischen Seele und Welt, zwischen Ich und Wirklichkeit, längst nicht mehr im heilen Raum der prästabilisierten Harmonie ab, sondern in den Labyrinthen der prästabilisierten Absurdität; und für Thomas Mann ist der einzig legitime, der gerade noch „unverteufelte“ literarische Ausdruck dieses Sachverhalts die tragische oder komische Parodie. ${ }^{23}$

Der letzte Satz ist besonders wichtig, denn folgt man diesem Gedanken bis in seine logischen Konsequenzen, so widerspricht er ja in gewissem Sinne den vorhergehenden Schlussfolgerungen. Die ,tragische Parodie“ bildet nämlich nicht den semantischen Kern des Romans, sondern ist nur eine literarische Ausdrucksform, die von Thomas Mann benutzt wird, um einen bestimmten „Sachverhalt" wiederzugeben. Die „prästabilisierte Absurdität“ des Seins sowie der tragische Bruch zwischen Ich und Welt gehören in den intratextuellen Raum von Leverkühn-Zeitblom, nicht aber in die extratextuelle Autorenperspektive, die auch dem aufmerksamen Leser zugänglich ist. Thomas Mann ist wesentlich ausgeglichener als seine Figuren, auch wenn er selbst seine emotionale Nähe zu Leverkühn betont: „Zeitblom ist eine Parodie meiner selbst. In Adrians Lebensstimmung ist mehr von meiner eigenen, als man glauben sollte“ ${ }^{، 24}$. Sogar wenn man dieses Autorenstatement für bare Münze nimmt (und da sollte man immer sehr vorsichtig sein), bleibt die Distanz zwischen dem Autor und seiner Romanwelt ungefähr so groß wie die zwischen den Leiden des jungen Werther und den Freuden des alten Goethe - und eben diese Abgrenzung ermöglicht das für die Modernisierung der Legende unentbehrliche Parodieausmaß. Eine ideale moderne Faust-Version ist weder Adrian Leverkühns Lebenswerk, das Oratorium Dr. Fausti Weheklag noch Serenus Zeitbloms Notizen, sondern ein komplexeres, mehrere Perspektiven umfassendes System - Thomas Manns Doktor Faustus als ein unzertrennbares, selbständiges Ganzes. (Daher kann man Milchs These, der Roman sei alles andere als eine „Faustdichtung unserer Tage ${ }^{625}$ schwer zustimmen.) Den Erzähler Zeitblom zitierend: ,[...] denn nur die Kunst könne einem Leben Schwere verleihen, das sonst so seiner Facilität sich zu Tode langweilen würde.“'26 Diese multiperspektivische „Schwere“ bedeutet aber nicht, dass im Roman bloß eine breite Palette verschiedener Kunstauffassungen präsentiert wird, nach dem Motto: man wähle, was man mag. Mehrere figureninkarnierte Ideen enthaltend (und sie bewusst überwindend) besitzt Doktor Faustus eine eigene, konkrete und

\footnotetext{
${ }^{23}$ Ebd., S. 325-326.

${ }^{24}$ Zitiert nach: Eike Midell: Thomas Mann. Versuch einer Einführung in Leben und Werk, Leipzig 1975, S. 249.

${ }^{25}$ Werner Milch: Thomas Manns „Doktor Faustus “, in: ders.: Kleine Schriften zur Literatur- und Geistesgeschichte, Heidelberg/Darmstadt 1957, S. 230.

${ }^{26}$ Mann: Doktor Faustus, S. 226.
} 
beinahe didaktische Aussage. Die belehrende Funktion der Kunst wird bei Mann permanent beibehalten. Zeitbloms Anschauungen zu diesem Thema scheint auch der Autor ganz und gar zu teilen:

[...] ja, mein Fragen, Sorgen und Bangen ging darüber hinaus und galt in der Stille meines Herzens geradezu der Legitimität seines Tuns, seinem zeitlichen Anrecht auf die Sphäre, in die er sich versenkte [...] kurz, es bestand in dem liebenden und angstvollen Verdacht eines Ästhetizismus, der meines Freundes Wort: das ablösende Gegenteil der bürgerlichen Kultur sei nicht Barbarei, sondern die Gemeinschaft, dem quälendsten Zweifel überlieferte.

Hier kann niemand mir folgen, der nicht die Nachbarschaft von Ästhetizismus und Barbarei, den Ästhetizismus als Wegbereiter der Barbarei in eigener Seele, wie ich, erlebt hat - der ich diese Not freilich nicht aus mir selbst, sondern mit Hilfe der Freundschaft für einen teueren und hochgefährdeten Künstlergeist erlebte. ${ }^{27}$

Ersetze man die Freundschaft mit dem „,hochgefährdeten Künstlergeist“ durch die langjährige ,Freundschaft' mit der literarischen Kunst - so könnten die oben zitierten Worte auch Zeitbloms Schöpfer zugeschrieben werden. Das über den Ästhetizismus gefällte Urteil bildet seinerseits eine perfekte Illustration zur ethischen Sendung von Doktor Faustus, die Hermann Kurzke, das Nietzsche-Motiv analysierend, pointiert auslegt:

Anders als die Sozialisten [...] sieht Mann in Nietzsche nicht den Verkünder, sondern den Seismographen des heraufziehenden Faschismus. Aber er spricht doch von zwei großen Irrtümern Nietzsches. Der erste sei, daß er das Machtverhältnis zwischen Instinkt und Intellekt verkannt habe, als sei der Geist wirklich das gefährlich Dominierende. Der zweite sei, daß er Leben und Moral in einen Gegensatz bringe, als sei die Moral lebensfeindlich. Sie sei vielmehr höchst lebensnützlich, und der wahre Gegensatz sei der von Ethik und Ästhetik, und nicht die Moral, sondern die Schönheit ist todverbunden. ${ }^{28}$

Die Dichotomie von Ethik und Ästhetik ist tatsächlich einer der zentralen Ideenkonflikte im Roman. Jedes Kunstwerk, wie ethisch makellos es auch sein mag, ist aber von Natur aus ein ästhetisches Phänomen. Betrachtet man den Ästhetizismus als eine moralisch gefährliche Einstellung, dann wird der Künstler (wie ihn Thomas Mann versteht) vor die entscheidende Frage gestellt: ob und wie können Ethik und Ästhetik in einer Art modernem Aufklärungsroman vereinbart werden? In allem, was der Text über die Kunst und ihre Aufgaben sagt, dient der Erzähler überwiegend als ein mehr oder weniger direkter Autorenvermittler. Serenus Zeitblom ist jedoch, wie bereits unterstrichen wurde, in der Rolle der Narrationsinstanz auf sämtlichen Ebenen höchst begrenzt. Er erkennt zwar das Problem, aber sein Versuch, sich damit auseinanderzusetzen, ist reich an Fehltritten. Sein Stil, den Heller „Thomas Manns eigenen Stil belächelnd und betrauernd ${ }^{\text {‘29 }}$ nennt, scheint zu altmodisch, pathetisch, unfreiwillig ko-

\footnotetext{
${ }^{27}$ Ebd., S. 507.

${ }^{28}$ Hermann Kurzke: Thomas Mann. Epoche - Werk - Wirkung, 2. überarbeitete Aufl., München 1991, S. 273.

${ }^{29}$ Heller: Thomas Mann. Der ironische Deutsche, S. 308.
} 
misch zu sein; seine Versicherungen, er schreibe keinen Roman, entpuppen sich als unglaubwürdige Mystifikationen; sein Anspruch auf ein tiefes Kunstverständnis wirkt nicht besonders überzeugend, da er selbst kein Künstler ist und dazu noch eine der Romanfiguren ist, weshalb seiner Position an der distanzierten Objektivität eines auktorialen Erzählers fehlt. Um es mit Orłowski auf den Punkt zu bringen:

Die Geschichte Serenus Zeitbloms ist die Geschichte des Versagens in zwiefacher Hinsicht: die Geschichte des handelnden Menschen und des interpretierenden Erzählers. ${ }^{30}$

Das Pharisäertum als Haltung im Leben (als literarischer Held) und im Informieren und Bewerten (als auktorialer Erzähler) ist dem Altphilologen eigen, und dies ist nur deshalb möglich, weil Zeitblom dem gedanklichen Prädestinationsmodell, dem fatalistischen Geschichtsauffassen folgt. ${ }^{31}$

Zurecht sieht Orłowski in Zeitblom einen unzuverlässigen Erzähler, dessen Aufgabe, Leverkühns Leben und Schaffen möglichst wahrheitsgetreu darzustellen, zum Scheitern verurteilt ist. Seine Person sowie seine Worte machen einen erstaunlich uneinheitlichen Eindruck, wenn man dahinter die außertextuelle Autoreninstanz nicht bemerkt. Im Gegenteil: Sieht man in Zeitblom einen funktionsaufgeladenen, prismatischen Teil der Erzählwelt, so ist die endgültige Botschaft des Textes leichter zu entziffern. Die Lösung, für die Doktor Faustus, etwas verschleiert, plädiert, ist zweifellos eine humanistisch-aufklärerische. Die Form, in die diese Lösung verpackt wird, geht dagegen von den Bedürfnissen und Erwartungen eines modernen, der „Dialektik der Aufklärung“" bewussten Rezipienten aus. Ähnlich wie Joseph und seine Brüder eine Humanisierung des Mythos ist, erfolgt in Doktor Faustus die Humanisierung der Legende. So entsteht eine eigenartige, scheinbar spielerische, in Wirklichkeit aber äuBerst ernstgemeinte Mischung aus einem autoreferenziellen ,Maskenball' und einem beharrlichen, obgleich absichtlich vertonten Appell an den Humanismus als treibende Kraft der sozial engagierten Kunst. Stilistisch innovativ und auch semantisch eher unerwartet ist dabei die Verknüpfung von modernen (ja die Postmoderne verkündigenden $^{32}$ ) narrativen Mitteln mit traditionellen Sprach-, Genre- und Gestaltungsmustern sowie ihre Verwendung zum Zwecke einer klassisch humanistischen Aufklärungssendung.

\section{Literatur}

Fix, Peter; Fix, Ulla; Hermsdorf, Klaus; Hofmann, Fritz; Matter, Henry; Paul, Konrad: Das erzählerische Werk Thomas Manns, Berlin/Weimar 1976.

\footnotetext{
${ }^{30}$ Orłowski: Prädestination des Dämonischen, S. 173.

${ }^{31}$ Ebd., S. 177.

${ }^{32}$ Jan-Dirk Müller sieht in Adrian Leverkühn den typischen Repräsentanten ,einer gerade einmal knapp zweihundert Jahre herrschenden Kunstphilosophie, wie die die Moderne und erst recht die Postmoderne verabschiedet hat“. Siehe in: Thomas Mann, Doktor Faustus, 1947-1997, hrsg. von Werner Röcke, Bern u.a. 2001, S. 169.
} 
Flaubert, Gustav: Madam Bovary, Berlin 1980.

Heller, Erich: Thomas Mann. Der ironische Deutsche, Frankfurt a.M. 1959.

Hilgers, Hans: Serenus Zeitblom: der Erzähler als Romanfigur in Thomas Manns „Doktor Faustus”, Frankfurt a.M. 1995.

Hilscher, Eberhard: Thomas Mann. Leben und Werk, Berlin 1983.

Jacquard, Jean-Philippe: Literatura kak takovaja [Literatur als solche], Moskva 2011.

Kurzke, Hermann: Thomas Mann. Epoche-Werk-Wirkung, 2. überarbeitete Aufl., München 1991.

Mann, Thomas: Doktor Faustus, 2. Auflage, Berlin/Weimar 1975.

Midell, Eike: Thomas Mann. Versuch einer Einführung in Leben und Werk, Leipzig 1975.

Milch, Werner: Thomas Manns „Doktor Faustus“, in: Milch, Werner: Kleine Schriften zur Literaturund Geistesgeschichte, Heidelberg/Darmstadt 1957.

Nabokov, Vladimir: Lekcii po zarubezhnoj literature [Vorlesungen über die fremdsprachige Literatur], Sankt-Petersburg 2011.

Neumann, Michael: Thomas Mann. Romane, Berlin 2001.

Orłowski, Hubert: Prädestination des Dämonischen. Zur Frage des bürgerlichen Humanismus in Thomas Manns „,Doktor Faustus“, Poznań 1968.

Schmidt-Schütz, Eva: „,Doktor Faustus “ zwischen Tradition und Moderne, Frankfurt a.M. 2003.

Stange, Carl R.: Thomas Manns „Doktor Faustus “, in: Literarische Revue 1948.

Thomas Mann, Doktor Faustus, 1947-1997, hrsg. von Werner Röcke, Bern u.a. 2001. 